\title{
Desempenho ocupacional de mulheres submetidas à mastectomia
}

\author{
Jamylle Silva de Brito ${ }^{a}$, Juliana Fonsêca de Queiroz Marcelino ${ }^{\mathrm{a}, \mathrm{b}}$ \\ aPrograma de Residência Multiprofissional Integrada em Saúde - PRMIS, Hospital das Clínicas, \\ Universidade Federal de Pernambuco - UFPE, Recife, PE, Brasil \\ bDepartamento de Terapia Ocupacional, Universidade Federal de Pernambuco - UFPE, Recife, PE, Brasil
}

\begin{abstract}
Resumo: Visto que dentre as diversas abordagens terapêuticas contra o câncer de mama a mastectomia é a mais temida entre as mulheres, devido às suas características invasivas e agressivas para o corpo feminino, além de suas repercussões biológicas, funcionais, emocionais e sociais, o presente estudo teve o objetivo de descrever o desempenho ocupacional de mulheres submetidas à mastectomia atendidas em um hospital escola da cidade do Recife, PE, a partir da autoavaliação do desempenho funcional e da satisfação delas em suas atividades de vida diária, produtiva e de lazer. Foi realizado um estudo transversal e descritivo com 21 mulheres, de junho a setembro de 2011, utilizando-se um questionário sociodemográfico e uma avaliação ocupacional por meio da Medida Canadense de Desempenho Ocupacional (COPM). A área de desempenho mais comprometida foi a produtiva, seguida do lazer. O estudo comprovou que os cuidados com o corpo após a mastectomia não configuram, nas mulheres operadas, impedimentos no desempenho de suas atividades, contudo são necessários rearranjos para manter uma performance satisfatória.
\end{abstract}

Palavras-chave: Câncer de Mama, Mastectomia, Terapia Ocupacional.

\section{Occupational performance of women subjected to mastectomy}

\begin{abstract}
Among the several therapeutic approaches to combat breast cancer, mastectomy is the most feared by women, not only because of its aggressive invasive characteristics to the female body, but also because of its biological, functional, emotional, and social repercussions. The objective of the present study was to outline the occupational performance profile through the description of daily life, productive and leisure activities of women that have undergone mastectomy. This is a descriptive cross-sectional study with 21 women carried out at a school hospital in Recife, Pernambuco state, Brazil, from June to September, 2011. We used a sociodemographic questionnaire and occupational assessment by the Canadian Model of Occupational Performance (CMOP). In the occupational performance evaluation, Productivity was the most compromised area, followed by Leisure. Taking care of the body after mastectomy was not a restraint to the development of the daily-life, productive and leisure activities for the operated women, although some reorganization was needed to maintain a satisfactory performance.
\end{abstract}

Keywords: Breast Cancer, Mastectomy, Occupational Therapy.

\section{Introdução}

O câncer de mama, dentre as neoplasias malignas que atingem as mulheres, tem sido o responsável pelos maiores índices de mortalidade no mundo, tornando-se uma das grandes preocupaçóes em saúde pública na assistência à saúde da mulher. Sua prevalência encontra-se mais marcante em mulheres na faixa etária entre 40 e 69 anos, superando apenas as mortes provocadas por doenças cardiovasculares e causas externas (BRASIL, 2009).

Nos países ocidentais, a neoplasia de mama está sendo considerada o tipo de câncer mais temido devido à sua alta frequência e aos seus extensos efeitos 
psicossociais (SILVA; ALBUQUERQUE; LEITE, 2010). Segundo dados do Ministério da Saúde, o Brasil, principalmente nas regiôes Nordeste, Sul e Sudeste, está entre os países com a mais elevada taxa de incidência de neoplasia de mama, refletindo as variaçóes decorrentes dos perfis heterogêneos de exposiçáo a fatores de risco e diferentes modos de vida (INSTITUTO..., 2008; DUARTE; ANDRADE, 2003).

O câncer de mama é o tipo mais comum entre as mulheres, respondendo por $22 \%$ dos casos novos a cada ano. Estimativas apontaram 52.680 novos casos para 2012 (INSTITUTO..., 2008). A explicação desse percentual, segundo o Instituto Nacional do Câncer, do Ministério da Saúde, está diretamente relacionada à maior exposição dos indivíduos a fatores de risco cancerígenos. Os atuais padrões de vida adotados em relação ao trabalho, nutrição e consumo em geral expóem os indivíduos a fatores ambientais mais agressivos, relacionados a agentes químicos, físicos e biológicos resultantes de um processo de industrializaçáo cada vez mais evoluído (BRASIL, 2009; INSTITUTO..., 2008).

A conduta de tratamento para a neoplasia é ampla e envolve uma série de intervençóes terapêuticas como quimioterapia, radioterapia, hormonioterapia e cirurgia (BERGMANN et al., 2007).

A terapêutica cirúrgica do câncer de mama é conhecida como mastectomia, a qual pode ter um formato conservador, com ressecção apenas de uma parte da mama chamada de quadrantectomia, ou ter o formato radical, com retirada total do órgão, acompanhado de linfadenectomia. Essa circuncisão cirúrgica está relacionada diretamente ao nível tumoral na qual a neoplasia foi diagnosticada (BREGNOL; DIAS, 2010).

Dentre as abordagens terapêuticas contra o câncer de mama, a extirpação cirúrgica do seio é considerada a mais temida entre as mulheres, devido às características invasivas e agressivas do procedimento para o corpo feminino, além das repercussóes biológicas, emocionais e sociais envolvidas no processo de amputação de um órgão símbolo da maternidade, socialmente ligada a um importante potencial erógeno (SILVA; ALBUQUERQUE; LEITE, 2010).

Apesar de ser considerado um agravo de bom prognóstico se diagnosticado e tratado com rapidez, a neoplasia possui ainda na sociedade um caráter estigmatizante, associado ao significado de fragilidade, limitação e morte, encarados tanto pela mulher acometida pela doença quanto por sua família e comunidade (INSTITUTO..., 2008; DUARTE; ANDRADE, 2003).
Dentre as manifestaçóes e as intercorrências consequentes a todo o seguimento terapêutico de quimioterapia, radioterapia e cirurgia contra o câncer de mama, os achados podem variar desde sinais e sintomas de alopecia, anemia, náuseas, vômitos e reaçóes de hipersensibilidade até consequências mais graves como disfunção cardíaca, infertilidade, menopausa precoce, erisipela, fibroses subcutâneas que expóem as mulheres ao risco de linfedema. Lesões no plexo braquial, neuropatias e limitação de amplitude de movimento do ombro homolateral à mastectomia também são possíveis, comprometendo substancialmente a mobilidade e funcionalidade do membro superior do lado da mama afetada. Achados como parestesias, dor localizada e/ou generalizada, como mialgias e artralgias, fadiga, falta de ar e insônia também não são queixas incomuns durante o prosseguimento do tratamento do câncer de mama (OLIVEIRA et al., 2010).

Nesse sentido, pode-se perceber que todos os procedimentos terapêuticos e as possíveis complicaçôes presentes no tratamento do câncer de mama, sejam elas imediatas ou tardias, culminam em sérios desconfortos e comorbidades físicas, emocionais e sociais, perturbando e colocando em risco temporário ou permanente o desempenho ocupacional das mulheres durante as atividades de vida diária, produtivas e de lazer (BREGNOL; DIAS, 2010).

O Desempenho Ocupacional, de acordo com a Occupacional Therapy Guidelines for Clientcentred Pratice da Associação Canadense de Terapia Ocupacional, define-se como a habilidade de realizar rotinas e desempenhar papéis e tarefas, envolvendo as áreas de autocuidado, produtividade e lazer em resposta às demandas do meio externo e interno ao indivíduo (LAW et al., 2009).

Nessa perspectiva, a Medida Canadense de Desempenho Ocupacional (COPM) foi desenvolvida em 1990 e publicada no ano seguinte, por pesquisadores canadenses, com o intuito de ser utilizada como guia por terapeutas ocupacionais, baseando-se na prática centrada no cliente.

A COPM é uma medida válida e confiável, que tem sido utilizada de forma bem-sucedida com diversos grupos de clientes, em diferentes países (CARSWELL, 2004; EYSSENET et al., 2005; PARKER; SYKES, 2006; CHATFIELD; BECKETT, 2007). Os estudos atestam sua versatilidade internacional e a aplicabilidade cultural, mesm0 tendo ela sido desenvolvida em contexto cultural canadense (CARSWELL, 2004; CHATFIELD; BECKETT, 2007). A COPM, desde sua publicação já havia sido traduzida, até o ano de 2005, para 24 idiomas diferentes, sendo utilizada 
por terapeutas ocupacionais em mais de 35 países ao redor do mundo (BASTOS; MANCINI; PYLÓ, 2010; MCCOLL et al., 2005). Nos últimos anos, o número de publicaçóes que utilizaram a COPM tem aumentado (LAW et al., 2009). Recentemente, a referida avaliação foi traduzida para a língua portuguesa e publicada no Brasil (LAW et al., 2009). No presente estudo, a sigla original em inglês será mantida, visto que a mesma é utilizada internacionalmente como indexador na área de Terapia Ocupacional.

A COPM tem por finalidade detectar a autopercepção de clientes com diferentes tipos de incapacidade em distintos estágios de vida (LAW et al., 2009). Ela considera o desempenho ocupacional como resultado de interação entre a pessoa, o ambiente, a ocupaçáo e a produção, bem como os componentes de desempenho (físico, mental, sociocultural e espiritual) e o ambiente, de acordo com o momento de desenvolvimento, os papéis ocupacionais e a motivação de cada sujeito (BASTOS; MANCINI; PYLÓ, 2010).

Partindo dessa premissa, o presente estudo teve como objetivo descrever o desempenho ocupacional de mulheres submetidas à mastectomia atendidas em um hospital escola da cidade do Recife, a partir de uma autoavaliação do seu desempenho funcional e de sua satisfação nas atividades de vida diária, produtivas e de lazer.

A opção por esse recorte se deu a partir do entendimento de que as atividades humanas se efetivam nessas três áreas da vida (vida diária, produtiva e lazer), sendo as repercussóes da doença oncológica responsáveis por mudanças transitórias e permanentes do desempenho ocupacional, capazes de comprometer a integridade física e psicossocial das mulheres tratadas cirurgicamente do câncer mamário.

Com isso surge a necessidade dos profissionais de saúde investigarem não somente as consequências físicas que determinada condição de saúde provoca, mas o impacto dessa condiçáo sobre a capacidade do indivíduo em desempenhar suas atividades desejadas e esperadas de forma satisfatória nos seus diferentes contextos ocupacionais.

\section{Metodologia}

Trata-se de um estudo transversal e descritivo, envolvendo 21 mulheres submetidas à mastectomia, na fase tardia, recrutadas no Hospital das Clínicas da Universidade Federal de Pernambuco (HC-UFPE), a partir da captação das mulheres nos ambulatórios de ginecologia e mastologia da referida instituição, no período de junho a setembro de 2011.

A referida amostra fez referência ao total de pacientes atendidas nos ambulatórios citados anteriormente que atenderam aos critérios de inclusão da pesquisa durante o período da coleta de dados. Os critérios de inclusão envolviam ser mulher com diagnóstico de câncer de mama na fase tardia, após procedimento de mastectomia unilateral, com classificação de Performance Status 0, ou seja, com capacidade de manter um desempenho funcional nas atividades cotidianas de forma considerada normal, acompanhada pelo Hospital das Clínicas.

Essa classificação conhecida como escala de medida da capacidade funcional de Perfomance Status (PS) consiste em um método de avaliação clínica de pacientes oncológicos, reconhecido pela OMS, classificado segundo o Eastern Cooperative Oncology Group (ECOG). A referida classificação tem uma variação de escores de 0 a 4 na qual PSO refere-se a atividade normal; PS1, a paciente com sintomas da doença mas que deambula e leva o seu dia normalmente; PS2, a paciente fora do leito mais de 50\% do tempo; PS3, a paciente acamada por mais de $50 \%$ do tempo, carente de cuidados mais intensivos; e PS4, a pacientes restritos ao leito (BRASIL, 2001).

A fase cirúrgica tardia, por sua vez, é o período que compreende do $12^{\circ}$ ao $18^{\circ}$ mês após o procedimento cirúrgico (SASAKI; LAMARI, 2007). Essa fase foi eleita como critério de inclusão para o estudo por tratar-se de um momento no qual a maioria das mulheres já se encontra bem adaptada às mudanças corporais e funcionais, sendo considerado o momento ideal para a realizaçáo de avaliaçôes referentes aos resultados definitivos da cirurgia de mastectomia (DUARTE; ANDRADE, 2003).

Foram excluídas da pesquisa as mulheres com déficit cognitivo ou perturbação mental que dificultasse o entendimento do processo metodológico; pacientes com diagnóstico de metástase; mulheres que realizaram mastectomia parcial, reconstrução mamaria ou encontravam-se submetidas ao tratamento de quimioterapia e radioterapia no momento da entrevista.

Os dados foram coletados pela própria pesquisadora, sendo utilizados como instrumentos um roteiro de entrevista estruturado, abrangendo questôes acerca dos dados sociodemográficos e clínicos, desenvolvido especialmente para o estudo, seguido da avaliaçáo funcional com a COPM, em sua versão brasileira traduzida, publicado por Law et al. (2009). 
A COPM é um instrumento de avaliação funcional padronizado e individualizado que pode ser útil para clientes com diferentes incapacidades, independentemente de diagnósticos específicos, sendo considerado um importante guia da prática clínica do terapeuta ocupacional (MCCOLL et al., 2005; PARKER; SYKES, 2006). Essa medida abrange três áreas de desempenho ocupacional: atividades de autocuidado (cuidados pessoais, mobilidade funcional e independência fora de casa), atividades produtivas (trabalho remunerado ou não, manejo das tarefas domésticas e escolares) e atividades de lazer (ação tranquila, recreação ativa e socialização) (LAW et al., 2009).

Essa avaliação de desempenho ocupacional conta com cinco passos: identificação de questôes no desempenho, classificação do grau de importância das atividades consideradas problemas, classificaçáo dos problemas em relação ao desempenho e à satisfação, acompanhado do cálculo da pontuação referente à avaliação inicial, seguido da reavaliação e, por fim, comparaçáo dos escores de mudança.

A partir do protocolo semiestruturado do COPM, a participante foi solicitada, inicialmente, a indicar tarefas funcionais que eram mais significativas para ela e cujo desempenho estava comprometido nas áreas de autocuidado, produtividade e lazer.

Em seguida, no passo dois, ocorreu a classificação da importância dessas atividades problemas, utilizando-se uma escala numérica de 1 a 10 . No passo três foram confirmados com a participante os cinco principais problemas e, em seguida, ela foi orientada a realizar uma autoavaliação quanto ao seu desempenho e sua satisfação em relação ao desempenho nas tarefas funcionais. Para isso, foi utilizado uma escala de variaçáo de pontos entre 1 e 10, onde a pontuação 1 foi considerada atividade cujo desempenho era incapaz de realizar e que gerava completa insatisfação, enquanto a pontuação 10 relacionava-se a um desempenho extremamente bom e que gerava excelente satisfação (CARSWELL, 2004). Dessa forma, quanto maior a pontuação, melhor a avaliação de desempenho e satisfação nas atividades funcionais citadas. De acordo com a avaliação da COPM, a partir da obtenção do escore 7 é sinalizado alteração de classificação de desempenho e satisfação, visto que a partir dessa pontuação passam a ser considerados moderados (LAW et al., 2009).

O quarto e o quinto passos da avaliação da COPM, que se referem à reavaliação e à pontuação dos escores de mudança, não constituíram parte do presente estudo, visto que não foi realizada nenhuma intervenção terapêutica ocupacional com as mulheres avaliadas, como previsto no projeto de pesquisa. Foi considerado que a aplicação dos três primeiros passos da COPM era suficiente para contemplar as informações necessárias, capazes de responder ao objetivo traçado para a pesquisa, de acordo com os princípios de análise permitidos no manual da referida medida de avaliaçấo e outras publicaçóes científicas na área que utilizavam a COPM (LAW et al., 2009).

Cada atividade problema referida pela população avaliada será apresentada pela estimativa dos escores indicados de desempenho e satisfação. Os dados foram organizados em planilha Excel $^{\bullet}$ e analisados com o programa SPSS versão 17.0.

Este estudo orientou-se pelos princípios éticos contidos na Resolução do Conselho Nacional de Saúde 196/96, que regem as diretrizes e normas regulamentadoras de pesquisas envolvendo seres humanos. Contou com a aprovação do Comitê de Ética e Pesquisa com Seres Humanos do Centro de Ciências da Saúde da Universidade Federal de Pernambuco, sob o registro CAAE: 0107.0.172.000-11 e colheu assinatura do Termo de Consentimento Livre e Esclarecido (TCLE) de todas as participantes.

\section{Resultados}

A distribuição das 21 pacientes de acordo com as variáveis sociodemográficas (Tabela 1) evidenciou que a maioria das mulheres submetidas à mastectomia em fase tardia da intervenção do Hospital das Clínicas de Pernambuco estão na faixa entre 50 e 59 anos (47,6\%), são procedentes da cidade do Recife $(52,4 \%)$, com ensino fundamental incompleto $(38,0 \%)$.

Nas variáveis referentes ao estado civil e número de filhos, respectivamente, $66,6 \%$ mantinham relação conjugal estável no momento da pesquisa, com média de 25 anos de uniáo, possuindo, a maioria $(52,2 \%)$, dois filhos na constituição familiar. No quesito religião, $85,8 \%$ das mulheres afirmavam ser praticantes de algum tipo de religiáo, dentre essas, $38,9 \%$ eram evangélicas.

Em relação ao desempenho de atividades produtivas (Tabela 2), 61,9\% das mulheres entrevistadas exerciam atividade profissional antes do diagnóstico de câncer de mama. Dentre as atividades pregressas citadas por elas estão: trabalhadora doméstica (24\%), professora (19\%), auxiliar de enfermagem $(14,3 \%)$, cozinheira $(14,3 \%)$, manicure $(14,3 \%)$, costureira $(9,5 \%)$ e copeira $(4,9 \%)$. Dentre elas, $61,6 \%$ mantinham ainda alguma atividade produtiva no momento da entrevista. Quando 
Tabela 1. Distribuição das pacientes submetidas à mastectomia atendidas no Hospital das Clínicas de Pernambuco, de acordo com as variáveis sociodemográficas, Recife, PE, 2011.

\begin{tabular}{|c|c|c|}
\hline VARIÁVEL & $N-21$ & $\%$ \\
\hline \multicolumn{3}{|l|}{ IDADE } \\
\hline $36-49$ & 03 & 14,3 \\
\hline $50-59$ & 10 & 47,6 \\
\hline Mais de 60 & 08 & 38,1 \\
\hline \multicolumn{3}{|l|}{ PROCEDÊNCIA } \\
\hline Recife/Região metropolitana & 11 & 52,4 \\
\hline Interior de Pernambuco & 10 & 47,6 \\
\hline \multicolumn{3}{|l|}{ ESCOLARIDADE } \\
\hline Analfabeto & 03 & 14,3 \\
\hline Ensino fundamental completo & 01 & 4,9 \\
\hline Ensino fundamental incompleto & 08 & 38,0 \\
\hline Ensino médio completo & 06 & 28,5 \\
\hline Ensino médio incompleto & 03 & 14,3 \\
\hline \multicolumn{3}{|l|}{ ESTADO CIVIL } \\
\hline Solteira & 01 & 4,8 \\
\hline Casada/União estável & 14 & 66,6 \\
\hline Divorciada & 01 & 4,8 \\
\hline Viúva & 05 & 23,8 \\
\hline \multicolumn{3}{|l|}{ FILHOS } \\
\hline Nenhum & 02 & 9,5 \\
\hline Um & 05 & 24,0 \\
\hline Dois & 11 & 52,2 \\
\hline Mais de dois & 03 & 14,3 \\
\hline \multicolumn{3}{|l|}{ PRÁTICA RELIGIOSA } \\
\hline Sim & 18 & 85,8 \\
\hline Não & 03 & 14,2 \\
\hline TIPO DE PRÁTICA RELIGIOSA & $\mathrm{N}-18$ & \\
\hline Católica & 06 & 33,3 \\
\hline Evangélica & 07 & 38,9 \\
\hline Espirita & 03 & 16,7 \\
\hline Outras & 02 & 11,1 \\
\hline
\end{tabular}

indagadas, 62,5\% delas referiram ter necessitado de mudança de função ou do tipo de atividade produtiva após o tratamento cirúrgico contra o câncer de mama. $\mathrm{Na}$ variável renda, a partir da amostra total de mulheres entrevistadas, 71,4\% possuíam algum tipo de rendimento, sendo $73,4 \%$ deles provenientes do trabalho assalariado, cujo valor manteve-se, na maioria das situaçôes (60\%), acima de um salário-mínimo.

Contudo, ao investigar as mulheres em relação ao seu rendimento familiar no momento anterior ao diagnóstico e tratamento do câncer de mama, $66,7 \%$ delas afirmaram ter havido mudança de renda, negativa para 53,3\% delas, caracterizando diminuição da renda.

No que diz respeito ao diagnóstico (Tabela 3), o mais encontrado entre as mulheres pesquisadas (57,2\%), segundo o Código Internacional de Doenças
Tabela 2. Distribuição das pacientes submetidas à mastectomia atendidas no Hospital das Clínicas de Pernambuco, de acordo com as variáveis relacionadas às atividades produtivas, Recife, PE, 2011.

\begin{tabular}{|c|c|c|}
\hline VARIÁVEL & & $\%$ \\
\hline $\begin{array}{l}\text { ATIVIDADE PROFISSIONAL } \\
\text { ANTES DO DIAGNÓSTICO } \\
\text { DE CANCER }\end{array}$ & $\mathrm{N}-21$ & \\
\hline $\operatorname{Sim}$ & 13 & 61,9 \\
\hline Não & 08 & 38,1 \\
\hline QUAL? & $\mathrm{N}-13$ & \\
\hline Trabalhadora doméstica & 05 & 24,0 \\
\hline Professora & 04 & 19,0 \\
\hline Auxiliar de enfermagem & 03 & 14,3 \\
\hline Cozinheira & 03 & 14,3 \\
\hline Manicure & 03 & 14,3 \\
\hline Costureira & 02 & 9,5 \\
\hline Copeira & 01 & 4,9 \\
\hline $\begin{array}{l}\text { MANTÉM O TRABALHO } \\
\text { APÓS A MASTECTOMIA }\end{array}$ & $\mathrm{N}-13$ & \\
\hline Sim & 08 & 61,6 \\
\hline Não & 05 & 38,4 \\
\hline $\begin{array}{l}\text { NECESSITOU MUDANÇA } \\
\text { DE FUNÇÃO? }\end{array}$ & N-08 & \\
\hline $\operatorname{Sim}$ & 05 & 62,5 \\
\hline Não & 03 & 37,5 \\
\hline $\begin{array}{l}\text { POSSUI RENDA } \\
\text { ATUALMENTE }\end{array}$ & $\mathrm{N}-21$ & \\
\hline Sim & 15 & 71,4 \\
\hline Não & 06 & 28,6 \\
\hline $\begin{array}{l}\text { QUAL TIPO DE RENDA } \\
\text { ATUAL? }\end{array}$ & $\mathrm{N}-15$ & \\
\hline Salário & 11 & 73,5 \\
\hline Autônoma & 02 & 13,3 \\
\hline Ajuda de parentes & 02 & 13,3 \\
\hline VALOR DA RENDA ATUAL & $\mathrm{N}-15$ & \\
\hline Um salário-mínimo & 06 & 40,0 \\
\hline Dois a três salários-mínimos & 09 & 60,0 \\
\hline $\begin{array}{l}\text { MUDANÇA DE } \\
\text { REDIMENTO APÓS } \\
\text { MASTECTOMIA }\end{array}$ & $\mathrm{N}-15$ & \\
\hline $\operatorname{Sim}$ & 10 & 66,7 \\
\hline Não & 05 & 33,3 \\
\hline $\begin{array}{l}\text { DIMINUIÇÃO DO } \\
\text { VALOR DA RENDA APÓS } \\
\text { MASTECTOMIA }\end{array}$ & $\mathrm{N}-15$ & \\
\hline $\operatorname{Sim}$ & 08 & 53,3 \\
\hline Não & 07 & 46,7 \\
\hline
\end{tabular}

(CID 10), foi a neoplasia mamária do tipo Carcinoma Ductal. Das mulheres entrevistadas, $71,4 \%$ foram submetidas à mastectomia da mama esquerda, com ocorrência de linfadenectomia em $66,7 \%$, no momento da cirurgia. Estavam em acompanhamento terapêutico há mais de ano, no HC-UFPE, 86,7\% das mulheres. 
Durante investigação da dominância lateral, foi constatado que $76,2 \%$ possuíam lado dominante direito. Em apenas 9,5\% dos casos avaliados, a dominância lateral (funcional) das mulheres era homolateral à mastectomia.

Todas as mulheres atendidas na referida instituição necessitaram de tratamento quimioterápico antes $(48,2 \%)$ ou após $(51,8 \%)$ a mastectomia, como complementação terapêutica. A maioria delas $(86,7 \%)$ submeteu-se, além de à quimioterapia, ao tratamento radioterápico. Quando indagadas sobre o conhecimento de antecedentes familiares para neoplasia, $42,9 \%$ afirmaram possuir casos de câncer na família.

$\mathrm{Na}$ avaliação do desempenho ocupacional, no primeiro passo da COPM, pôde-se constatar que a área de desempenho citada como mais comprometida pelas mulheres atendidas pelo HC-UFPE e participantes da pesquisa foi a área produtiva, seguida, em frequência, pelas áreas de lazer e de autocuidado.

Tabela 3. Distribuição das pacientes submetidas à mastectomia atendidas no Hospital das Clínicas de Pernambuco, de acordo com os dados clínicos, Recife, PE, 2011.

\begin{tabular}{lcc}
\hline \multicolumn{1}{c}{ VARIÁ VEL } & N- 21 & $\%$ \\
\hline $\begin{array}{l}\text { DIAGNÓSTICO PRINCIPAL } \\
\text { Carcinoma Lobular }\end{array}$ & 09 & 42,8 \\
Carcinoma Ductal & 12 & 57,2 \\
MASTECTOMIA & & \\
Direita & 06 & 28,6 \\
Esquerda & 15 & 71,4 \\
LINFADENECTOMIA & & \\
Sim & 14 & 66,7 \\
Não & 07 & 33,3 \\
DOMINÂNCIA LATERAL & & \\
Direita & 16 & 76,2 \\
Esquerda & 05 & 23,8 \\
TEMPO DE & & \\
ACOMPANHAMENTO & & \\
12 meses & 02 & 13,3 \\
12 a 18 meses & 19 & 86,7 \\
TRATAMENTOS A QUE FOI & & \\
SUBMETIDA & & \\
Quimioterapia & 21 & 100,0 \\
Radioterapia & 19 & 86,7 \\
Hormonioterapia & 07 & 33,1 \\
Reabilitação física (Fisioterapia) & 06 & 28,6 \\
HISTÓRIA DE CÂNCER NA & & \\
FAMILIA & & \\
Sim & 09 & 42,9 \\
Não & 07 & 33,1 \\
Não sabe informar & 05 & 24,0 \\
\hline
\end{tabular}

No total, 14 atividades foram apontadas pelos sujeitos da pesquisa como problema para o seu desempenho ocupacional (Tabela 4).

Durante a classificação da importância dessas atividades, as tarefas referidas foram: manter domicílio $(50,0 \%)$, manter relação sexual $(42,8 \%)$, retornar ao trabalho $(42,8 \%)$, conduzir veículos $(35,7 \%)$ manter-se no emprego (35,7\%), escrever e digitar (35,7\%), utilizar transporte coletivo - ônibus $(35,7 \%)$, lavar roupas $(28,6 \%)$, fazer compras $(28,6 \%)$, realizar passeios com a família $(28,6 \%)$, participar de atividades sociais $(28,6 \%)$, preparar alimentos $(21,4 \%)$, praticar atividade física $(21,4 \%)$ e realizar atividades artesanais (14,3\%).

Em relação aos escores de avaliação do grau de desempenho e satisfação obtidos no terceiro passo da COPM (Tabela 5), dentre as atividades de autocuidado referidas como importantes, porém com problemas de desempenho, $28,6 \%$ das mulheres entrevistadas apontaram bom desempenho (escore 8) em escrita e digitaçáo, contudo satisfação regular no que diz respeito a sua performance (escore 6). A Tabela 6 apresenta a classificação de desempenho e satisfaçáo considerados pela COPM.

No quesito relação sexual, a avaliação do desempenho foi indicada com dificuldade moderada (escore 6), refletindo-se durante autoavaliação em escore de pouca satisfação (escore 4) para $48 \%$ das mulheres avaliadas.

No quesito mobilidade funcional, usar o transporte coletivo (ônibus) foi avaliado por 19,1\% das mulheres como atividade de bom desempenho (escore 8), com

Tabela 4. Atividades problema apontadas pelas mulheres submetidas à mastectomia atendidas no Hospital das Clínicas de Pernambuco, Recife, PE, 2011.

\begin{tabular}{lcc}
\hline \multicolumn{1}{c}{$\boldsymbol{A T I V I D A D E S}$} & $\boldsymbol{F}$ & $\boldsymbol{\%}$ \\
\hline Manter domicílio & 07 & 50,0 \\
Manter relação sexual & 06 & 42,8 \\
Retornar ao trabalho & 06 & 42,8 \\
Conduzir veículos & 05 & 35,7 \\
Manter-se no emprego & 05 & 35,7 \\
Escrever/digitar & 05 & 35,7 \\
Utilizar transporte coletivo & 05 & 35,7 \\
Lavar roupas & 04 & 28,6 \\
Fazer compras & 04 & 28,6 \\
Realizar passeios com a família & 04 & 28,6 \\
Participar de atividades sociais & 04 & 28,6 \\
Preparar alimentos & 03 & 21,4 \\
Praticar atividade física & 03 & 21,4 \\
Realizar atividades artesanais & 02 & 14,3 \\
\hline F: Frequência em que a atividade foi citada durante avaliação \\
com COPM.
\end{tabular}


Tabela 5. Pontuação total referente à autoavaliação do desempenho e da satisfação nas atividades referidas como problema pelas mulheres submetidas à mastectomia atendidas no Hospital das Clínicas de Pernambuco, durante aplicação da COPM.

\begin{tabular}{|c|c|c|}
\hline PARTICIPANTE & $\begin{array}{c}\text { PONTUAÇÃO DO } \\
\text { DESEMPENHO }\end{array}$ & $\begin{array}{c}\text { PONTUAÇÃO DA } \\
\text { SATISFAÇÃO }\end{array}$ \\
\hline $\mathrm{A}$ & 6,0 & 5,0 \\
\hline B & 6,4 & 5,0 \\
\hline C & 6,4 & 5,0 \\
\hline D & 7,0 & 5,5 \\
\hline E & 7,2 & 5,7 \\
\hline F & 7,2 & 5,7 \\
\hline G & 7,8 & 6,2 \\
\hline $\mathrm{H}$ & 8,0 & 6,3 \\
\hline I & 8,2 & 6,4 \\
\hline $\mathrm{J}$ & 8,3 & 6,4 \\
\hline K & 8,4 & 6,5 \\
\hline $\mathrm{L}$ & 8,4 & 6,5 \\
\hline $\mathrm{M}$ & 8,5 & 6,5 \\
\hline $\mathrm{N}$ & 8,5 & 6,8 \\
\hline $\mathrm{O}$ & 8,7 & 6,8 \\
\hline $\mathrm{P}$ & 8,7 & 7,0 \\
\hline $\mathrm{Q}$ & 8,8 & 7,0 \\
\hline $\mathrm{R}$ & 8,8 & 7,2 \\
\hline $\mathrm{S}$ & 8,9 & 7,4 \\
\hline $\mathrm{T}$ & 9,0 & 7,7 \\
\hline U & 9,0 & 8,0 \\
\hline
\end{tabular}

Tabela 6. Classificação de desempenho e satisfação considerados pela COPM.

\begin{tabular}{|c|c|c|}
\hline ESCORE & DESEMPENHO & SATISFAÇÃO \\
\hline 01 & Incapaz de realizar & Completamente insatisfeito \\
\hline 02 & Realiza mas precisa de muita ajuda & Nada satisfeito \\
\hline 03 & Realiza mas precisa de ajuda & Muito pouco satisfeito \\
\hline 04 & Realiza com muita dificuldade & Pouco satisfeito \\
\hline 05 & Realiza com dificuldade & Baixa satisfação \\
\hline 06 & Realiza com dificuldade moderada & Regularmente satisfeito \\
\hline 07 & Realiza moderadamente bem & Moderadamente satisfeito \\
\hline 08 & Realiza bem & Satisfeito \\
\hline 09 & Realiza muito bem & Muito satisfeito \\
\hline 10 & Realiza extremamente bem & Extremamente satisfeito \\
\hline
\end{tabular}

regular satisfação (escore 6). Dirigir veículos foi avaliado como atividade de desempenho considerado moderadamente bom (escore 7) e de baixa satisfação (escore 5) em 37,5\% das situaçóes.

Dentre as atividades relacionadas à produtividade, no quesito tarefas domésticas foram encontradas as seguintes avaliaçóes: manter o domić́lio, citado por $70,4 \%$ como de desempenho moderadamente bom (escore 7) e de satisfação regular (escore 6); enquanto a atividade de fazer compras apresentou para 24,3\% escore de desempenho bom (8) e de baixa satisfação (escore 5); lavar roupas $(38,4 \%)$ apresentou desempenho moderadamente bom (escore 7), com regular satisfação (escore 6); enquanto, preparar alimentos $(17,9 \%)$ foi avaliada como atividade de bom desempenho (escore 8) e satisfação regular (escore 6).

Nas atividades de trabalho, o retorno para as atividades produtivas desenvolvidas anteriormente ao diagnóstico e ao tratamento do câncer de mama foi apontado por $63,3 \%$ das mulheres como atividade com desempenho de dificuldade moderada (escore 6) e de muito pouca satisfação (escore 3). Contudo, manter-se no emprego foi apontado por 35,2\% das mulheres como atividade de bom desempenho (escore 8), contudo de baixa satisfação (escore 5).

No quesito atividades de lazer, foram encontrados os seguintes achados: $39 \%$ realizam passeios com a família, avaliando seu desempenho como moderadamente bom (escore 7), acompanhado de 
satisfação regular (escore 6). Nas atividades sociais, 43,2\% participam com dificuldade moderada (escore 6) e baixa satisfação (escore 5).

Durante as entrevistas as mulheres referiram necessidade de participar das atividades/encontros em família como forma apenas de responder às exigências dos mesmos, e exercer o papel de mãe, esposa/ companheira, filha e amiga. Dentre as atividades sócias, a prática religiosa foi a mais citada $(85,8 \%)$ entre as mulheres avaliadas, seguida da prática de visitar amigos e parentes $(68,2 \%)$.

Ainda durante a autoavaliação das atividades de lazer, as mulheres submetidas à mastectomia, apontaram, para as atividades artesanais $(28,7 \%)$ e atividades físicas (16,9\%), um desempenho moderadamente bom (escore 7), porém de baixa satisfaçăo (escore 5).

\section{Discussão}

Nas mulheres brasileiras, a neoplasia mamária é o tipo de câncer que mais causa morte, porém se detectado em fase inicial, há altas chances de cura. Muitas vezes, ele é descoberto tardiamente, gerando tratamentos mutilantes com importantes repercussốes na vida da mulher, de sua família e da sociedade em geral (MISTURA; CARVALHO; SANTOS, 2011).

No presente estudo, a maior ocorrência de mastectomia no formato radical esteve entre as participantes na faixa etária de 50 a 59 anos, com ensino médio incompleto, em união conjugal estável, com dois filhos na constituição familiar, semelhantes às referências encontradas em outros estudos nacionais de coorte realizados com população feminina com câncer de mama (LEITE et al., 2012).

A maioria das participantes do estudo foi submetida no mínimo a dois tipos de tratamento (cirurgia e quimioterapia). Em pesquisa desenvolvida sobre a assistência oncológica pelo Sistema Único de Saúde a mulheres com câncer de mama realizada por Mistura, Carvalho e Santos (2011), verificou-se que a maioria as mulheres realizaram quimioterapia e tratamento local (cirurgia) como terapêutica contra o câncer, ocorrência observada também no presente estudo.

A dificuldade de acessibilidade aos serviços de saúde, a precariedade de métodos para detecção e rastreamento do câncer de mama, principalmente em hospitais públicos, dificultam a detecção precoce da doença, refletindo em diagnósticos mais graves e com maior grau de comprometimento (MANUEL et al., 2010).
Assim, vale enfatizar que, frente ao diagnóstico e às repercussóes do tratamento do câncer de mama, muitas vezes detectados em estado avançado, a mulher encontra-se vulnerável para o desenvolvimento de sentimentos como medo, tristeza e negação, associados a vários significados distintos, predominando a ameaça a seu símbolo de feminilidade e sensualidade, além das implicaçóes para sua vida cotidiana (ANDOLFATO; MARIOTTI, 2009).

Pela descrição das atividades citadas pela população entrevistada na presente pesquisa, os resultados sugerem que as limitaçóes apresentadas pelas mulheres pós-mastectomia geram dificuldades que influenciam não apenas a sua rotina diária mas também a sua permanência em atividades produtivas e o seu contato social de maneira satisfatória e prazerosa.

Segundo Silva e Riul (2011) e Dornelas e Galvão (2007), a maneira como as mulheres desempenhavam suas funções anteriormente ao diagnóstico do câncer influencia diretamente a maneira como desenvolverão e manterão o desempenho em suas atividades após o tratamento do câncer.

A necessidade em modificar e adaptar grande parte das atividades cotidianas vislumbrando um desempenho funcional o mais próximo possível $\mathrm{da}$ performance anterior ao processo de adoecimento gera, por vezes, incômodo e insatisfação entre as mulheres tratadas do câncer de mama, diante das novas necessidades de cuidados e novos limites do corpo (CHANÁ; ALBUQUERQUE, 2006).

A realização das atividades de vida diária (AVD), além de reforçar o senso de capacidade para o autocuidado das mulheres mastectomizadas, também possibilita a elas, o sentimento de satisfação pessoal e a oportunidade de escolhas (CAETANO; GRADIM; SANTOS, 2009).

Para Mello e Mancini (2007), a realização dessas atividades está associada ao estilo de vida de cada mulher, assim como a suas características de personalidade, valores pessoais, crenças e possibilidades de autocontrole.

Contudo, uma importante reflexão, nesse sentido, é trazida pelos autores supracitados, ao referirem que no processo saúde-doença estão presentes os contextos culturais e as diversas formas de dominação que conferem especificidades ao adoecimento das mulheres, assim como às determinaçóes de gênero. Esse olhar possibilita observar para além do fenômeno biológico da doença, integrando também aspectos sociais.

Assim, possibilita-se a compreensão do processo de adoecimento dessas mulheres pelas condiçóes de 
vida, de empobrecimento contínuo, de precarização das condiçôes de trabalho e de dificuldade de acesso aos serviços prestados pelo Sistema de Saúde de forma eficiente e efetiva (BITTENCOURT; SOUZA; MENEZES, 2010).

Vivenciar uma situação de doença como o câncer é uma experiência complexa e sofrida para qualquer pessoa. Ela traz consigo uma série de implicaçóes físicas, emocionais, sociais e econômicas para a vida dos sujeitos enfermos e sérias atribulaçóes para as famílias e toda a sociedade (BITTENCOURT; SOUZA; MENEZES, 2010).

As repercussóes do tratamento do câncer de mama comprometem o cotidiano das pessoas, impactando, as mudanças, na dinâmica pessoal e familiar, nas relações de trabalho e nas relaçóes sociais (CAETANO; GRANDIM; SANTOS, 2009).

Para Spalla e Santo (2009), a ausência de mecanismos de proteção e de suporte social, dentre elas a prática religiosa, compromete diretamente o desempenho funcional, assim como o sentimento de satisfação das mulheres mastectomizadas em seus diferentes contextos de vida.

O processo de continuidade das atividades relacionadas ao autocuidado, produtividade e de lazer, mesmo que de forma insatisfatória, sinaliza para as mulheres a possibilidade de recuperação do senso de utilidade ao provarem para si e para os outros ainda serem capazes de executar suas tarefas cotidianas, apesar das limitaçóes físicas, da fadiga, da necessidade de descanso e de cuidados (SILVA; ALBUQUERQUE; LEITE, 2010).

Dentre as atividades de autocuidado citadas como problemas, a manutenção do desempenho sexual foi bastante referida pelas mulheres, devido ao desinteresse delas na realização do ato sexual. Duarte e Andrade (2003) relatam que a diminuição da libido associada à sensação de fadiga são queixas comuns entre as mulheres após o processo da mastectomia (BITTENCOURT; SOUZA; MENEZES, 2010).

Esses sintomas, segundo Panobianco e Mamede (2002), podem ser atribuídos a causas fisiológicas e emocionais, como fadiga, ansiedade e estresse emocional provenientes do processo de adoecimento e do tratamento, assim como do medo da morte e, ainda, do comprometimento da imagem corporal da mulher mastectomizada.

Em relação ao desempenho das atividades relacionadas à produtividade, o grupo pesquisado apresentou escores de desempenho maiores do que os escores relacionados à satisfação. Dentre as situaçóes referidas como barreiras para o desempenho do trabalho remunerado, segundo Silva e Riul (2011), estáo: a necessidade de mudança da função produtiva desempenhada antes do diagnóstico e do tratamento do câncer de mama e os cuidados requeridos pela nova situação de saúde.

Contudo, o processo de continuidade das atividades produtivas sinalizou, para as mulheres entrevistadas, a possibilidade do senso de utilidade, apesar das limitações físicas, da sensação de fadiga, da necessidade de descanso e de cuidados específicos referidos por elas após a mastectomia.

Dentre os cuidados necessários, recomendados por Manuel et al. (2010) para as mulheres mastectomizadas estão: atentar quanto à exposição a temperaturas elevadas, assim como para queimaduras e cortes, evitar exceder-se em esforços físicos e movimentos repetitivos, como forma de prevenir o desenvolvimento de linfedema do membro superior. Esses cuidados, entretanto, não implicam, necessariamente, na cessação das atividades produtivas, porém requerem um olhar mais cuidadoso da mulher sobre o modo como são executadas, assim como atençáo aos contextos nos quais são praticadas (CAETANO; GRANDIM; SANTOS, 2009).

Contudo, os autores supracitados referem que não é incomum a diminuição do status econômico e da renda familiar após um diagnóstico de câncer, situação associada à redução do desempenho de atividades remuneradas, diante da crença pessoal, familiar e social de incapacidade para o trabalho.

Devem ser consideradas atividades produtivas e de trabalho, por definição, todas aquelas tarefas voltadas para a produção ou remuneraçáo, incluindo-se as náo assalariadas que contribuem para a subsistência dos indivíduos, a exemplo dos afazeres domésticos e/ou dos cuidados com familiares. Nesse sentido, pode-se entender que as atividades produtivas não se configuram somente como trabalho remunerado, embora o incluam, pois são consideradas genericamente como atividades que fazem as pessoas sentirem-se úteis em diferentes contextos (KEBBE, 2006; MANUEL et al., 2010; TOLDRÁ et al., 2010).

Algumas mulheres mantêm o seu desempenho produtivo, esforçando-se sem ajuda, embora com descontentamento, na tentativa de manter o poder construído e exercido por elas, na internalidade do seu espaço produtivo (CARDOSO; OLIVEIRA, 2004). De acordo com Carswell (2004), os indivíduos buscam aprender a estrutura da interação produtiva e social a que pertencem, a fim de reformularem uma conduta, possibilitando, assim, minimizar a exposição de um estigma. 
Contudo, é importante que todos os atores envolvidos nessa problemática (mulher, família, equipe de saúde e sociedade) considerem as repercussóes físicas reais inerentes ao processo de mastectomia, acompanhado ou não de linfadenectomia, relacionando-as à dominância lateral pregressa da mulher. Todos esses fatores devem ser respeitados e avaliados durante uma avaliação funcional envolvendo as áreas de autocuidado, produtividade e lazer. No presente estudo, a maioria da amostra da populaçáo selecionada $(90,5 \%)$ foi submetida a mastectomia contralateral ao da dominância, fato que deve ser considerado durante análise de todos os resultados.

$\mathrm{Na}$ área de desempenho do lazer, a maioria das mulheres participantes da pesquisa mencionou dificuldade para manter um processo de socialização satisfatório. Para Spalla e Santo (2009), esse fato pode ser confirmado pelo fato de muitas mulheres após tratamento oncológico manterem suas atividades de lazer apenas para a manutenção de seu papel social.

A socialização entre amigos e familiares pode ser um facilitador no processo de enfretamento e recuperação da doença. As mulheres que apresentam dificuldade para compartilhar com sua rede social a sua condição de saúde acabam por reforçar a própria expectativa negativa de estigmatizaçáo e incapacidade envolvida no imaginário social da doença oncológica (SILVA; ALBUQUERQUE; LEITE, 2010).

Para Kebbe (2006), o incômodo gerado pela percepçáo da falta de uma parte do corpo (a mama) revela nas mulheres a introjeção de valores históricos culturalmente atribuídos à simetria do corpo, relacionada, como sinônimo, a beleza, integridade física e funcionalidade, tornando-as, por ora, menos responsivas às expectativas culturalmente valorizadas relacionadas à aparência do corpo feminino.

O aumento da preocupação com o corpo, considerado frágil e agora requerente de constantes cuidados, assim como a necessidade de isolamento social, a desmotivação, a negativa de exposição do corpo "imperfeito" e a preocupaçáo com a aparência física podem ser considerados fatores limitantes do desempenho das atividades de lazer entre as mulheres mastectomizadas (KEBBE, 2006).

As alteraçôes no desempenho de atividades de lazer diante das limitaçóes físicas podem acarretar sentimentos depreciativos, interferindo na valorização pessoal de cada mulher, levando-as a vivenciar o processo de estigmatizaçáo e incapacidade sem perspectiva de retorno aos padróes anteriores de atividade (DORNELAS; GALVÃO, 2007).

Com isso, é relevante considerar que a fragilidade representada pela falência do corpo é minorada quando a mulher pode optar por recobrar sua autonomia e, com ela, seu autocontrole, seus desejos, sua história e seu corpo, desconstruindo o rótulo estigmatizante de incapacidade conferido pelos próprios familiares e pela sociedade (ANDOLFATO; MARIOTTI, 2009; BITTENCOURT; SOUZA; MENEZES, 2010).

Os achados desta pesquisa indicam, contudo, que os cuidados necessários com o corpo após processo cirúrgico da mastectomia não configuram, nas mulheres operadas avaliadas, impedimentos ao desempenho das atividades relacionadas ao autocuidado, produtividade e de lazer, pois todas mostraram-se capazes de manter a autonomia e a independência em seus diferentes contextos de vida. É importante lembrar, porém, que esse resultado pode ser explicado pelo período de investigação no qual o estudo se realizou, além do achado de que a mastectomia, para a maior parte das mulheres entrevistadas, ocorreu do lado contralateral ao da sua dominância funcional.

Entretanto, pôde ser detectado com a presente investigação que apesar de as mulheres avaliadas serem consideradas capazes de manter as suas atividades cotidianas de forma normal, foram necessários rearranjos no desempenho da maioria dessas atividades no momento pós-mastectomia, no qual muitas delas não foram capazes de manter uma performance satisfatória, tendo demonstrado durante a entrevista sentimentos de incômodo frente às mudanças de conduta e comportamento requeridos pela nova situação de saúde.

Evidencia-se, assim, a importância de investigar aspectos singulares envolvidos no processo de desempenho ocupacional de cada sujeito, situação permitida com o uso da COPM.

Desse modo, é imprescindível um olhar cuidadoso de uma equipe de saúde com caráter multiprofissional e interdisciplinar. Essas intervençôes têm como objetivo fazer uma junção entre conhecimentos e disciplinas que intercedam efetivamente na qualidade de vida das mulheres mastectomizadas, durante e após o tratamento, favorecendo de forma prioritária o seu retorno social e produtivo (OTHERO, 2010).

O terapeuta ocupacional, quando inserido na equipe de reabilitação oncológica, em especial no cuidado de mulheres submetidas à mastectomia, tem por objetivo tratar os agravos físicos, sensoriais e emocionais decorrentes de todo o tratamento.

A ação desse profissional visa resgatar a independência, a autonomia, a autoestima e a inclusão social desses sujeitos, auxiliando a mulher na compreensão de sua situação atual de saúde, desenvolvendo junto a elas estilos adaptativos de vida, 
integrando as capacidades funcionais e ocupacionais de forma prazerosa e satisfatória.

\section{Considerações finais}

As mudanças no desempenho ocupacional podem representar para as mulheres sentimento de incapacidade, considerando a funcionalidade não apenas em relação ao corpo físico mas também na relaçáo desse corpo com um mundo produtivo e social em que são depositadas expectativas.

Diante do presente estudo, pôde-se verificar que as dificuldades presentes no desempenho ocupacional das mulheres participantes desta pesquisa estáo de acordo, em grande parte, com aquelas descritas pela literatura.

A COPM mostrou ser um instrumento de avaliação útil e clinicamente aceitável, visto que permitiu identificar as áreas de desempenho ocupacional comprometidas, demonstrando a satisfação das mulheres submetidas à mastectomia em relação a sua performance ocupacional.

O uso da abordagem centrada no cliente, modelo teórico sob o qual a COPM foi desenvolvida, permitiu o envolvimento dos sujeitos no seu processo de avaliação funcional, possibilitando durante o seu desenvolvimento a orientação das mulheres avaliadas quanto às adaptaçôes das atividades de autocuidado, produtivas e de lazer, para a promoçáo de uma vida saudável e prazerosa, em seus diferentes contextos.

Observa-se, contudo, a necessidade de novas pesquisas utilizando a escala de avaliaçáo de desempenho ocupacional, COPM, com a população em estudo, nas quais seja possível o desenvolvimento de um plano de intervençâo terapêutico ocupacional, permitindo com isso o cálculo de diferença dos escores de mudanças de desempenho e satisfação das mulheres mastectomizadas em suas atividades de vida diária, produtiva e de lazer, antes e depois da intervenção.

Dessa forma será possível identificarem-se os ganhos e as limitaçóes do tratamento terapêutico ocupacional junto à equipe de saúde, para a promoçáo, a recuperação e a reabilitação física, mental e social dessas mulheres, contribuindo assim para uma reinserção familiar e comunitária de configuração eficiente e eficaz.

Nesse sentido é estratégico viabilizar condições de acesso da população brasileira a serviços de saúde estruturados, solidificando uma rede assistencial integral e interdisciplinar, garantindo o acesso das mulheres a consultas e exames diagnósticos eficazes, capazes de diminuir a crescente incidência de casos avançados de câncer de mama na população feminina e a ocorrência de tratamentos mutilantes, cujas consequências repercutem diretamente sobre a vida pessoal, familiar e social delas.

Contudo, é importante considerar que o contexto no qual as equipes de saúde estão inseridas permanece, em sua totalidade, saturado de determinações (econômicas, políticas, culturais, ideológicas) que exigem mais do que açōes imediatas, instrumentais e de cunho psicossocial, mas implicam intervençôes que emanem de escolhas, que passem pelos condutos de uma prática baseada na razão crítica e no desejo dos sujeitos, a fim de estabelecer maior participação e satisfação deles com o seu próprio processo de tratamento e reabilitaçáo psicossocial.

Quando inserido na equipe de reabilitaçáo, o terapeuta ocupacional está habilitado, nesse contexto, a identificar as necessidades da mulher, seus sintomas, suas causas e o impacto desses no seu cotidiano, promovendo o seu desempenho funcional no nível máximo, estimulando a independência, a autonomia e a qualidade de vida de todos os sujeitos envolvidos no processo.

Torna-se, desse modo, um grande desafio responder às singularidades demandadas da experiência de uma doença como o câncer e entender que as necessidades individuais expressam, ao mesmo tempo, um caráter coletivo. São imperiosas a articulação e a discussão sobre as determinaçôes estruturais das demandas das usuárias do serviço e das equipes de saúde para além da doença, e que se expressam na singularidade da vida dos sujeitos e de seus familiares.

\section{Referências}

ANDOLFATO, C.; MARIOTTI, M. C. Avaliação do paciente em hemodiálise por meio da medida canadense de desempenho ocupacional. Revista de Terapia Ocupacional da Universidade de São Paulo, São Paulo, v. 20, n. 1, p. 1-7, 2009.

BASTOS, S. C. A.; MANCINI, M. C.; PYLÓ, R. M. $\mathrm{O}$ uso da medida canadense de desempenho ocupacional (COPM) em saúde mental. Revista de Terapia Ocupacional da Universidade de São Paulo, São Paulo, v. 21, n. 2, p. 104-110, 2010.

BERGMANN, A. et al. Incidência e prevalência de linfedema após tratamento cirúrgico do câncer de mama: revisão de literatura. Revista Brasileira de Cancerologia, Rio de Janeiro, v. 53, n. 4, p. 461-470, 2007.

BITTENCOURT, J. F. V.; SOUZA, I. C. T. C.; MENEZES, M. F. B. A mulher submetida à mastectomia: tecendo possibilidades do cuidar em enfermagem considerando o apoio da rede social primária. Revista Brasileira de Cancerologia, Rio de Janeiro, v. 56, n. 2, p. 269, 2010. 
BRASIL. Ministério da Saúde. Instituto Nacional de Câncer. Cuidados paliativos oncológicos: controle da dor. Rio de Janeiro: Ministério da Saúde, 2001.

BRASIL. Ministério da Saúde. Instituto Nacional de Câncer. Estimativa 2010: incidência de câncer no Brasil. Rio de Janeiro: Ministério da Saúde, 2009.

BREGNOL, R. K.; DIAS, A. S. Alterações funcionais na linfadenectomia. Revista Brasileira de Cancerologia, Rio de Janeiro, v. 56, n. 1, p. 25-33, 2010.

CAETANO, E. A.; GRADIM, C. V. C.; SANTOS, L. E. S. S. Câncer de mama: reaçôes e enfrentamentos ao receber o diagnóstico. Revista Enfermagem UERJ, Rio de Janeiro, v. 17, n. 2, p. 257-261, 2009.

CARDOSO, F. R.; OLIVEIRA, A. L. E. A. S. Avaliação postural em mulheres submetidas à mastectomia modificada: estudo de cinco casos. Revista Latinoamericana de Mastologia, Rio de Janeiro, v. 5, n. 1, p. 13-18, 2004.

CARSWELL, A. Prática baseada no cliente na terapia ocupacional: guia para a implementação. São Paulo: Roca, 2004.

CHANÁ, P.; ALBUQUERQUE, D. La Clasificación Internacional del Funcionamiento, de la Discapacidad y de la Salud (CIF) y la práctica neurológica. Revista Chilena de Neuro-psiquiatria, Santiago, v. 44, n. 2, p. 89-97, 2006.

CHATFIELD, J.; BECKETT, D. The canadian occupational performance measure: use in an independent living centre. International Journal of Therapy and Rehabilitation, London, v. 14, n. 6, p. 280-284, 2007. http://dx.doi.org/10.12968/ijtr.2007.14.6.23900

DORNELAS, A.; GALVÃO, C. Avaliação das habilidades psicossociais e componentes psicológicos. In: CAVALCANTI, A.; GALVÃO, C. Terapia Ocupacional: fundamentos e prática. Rio de Janeiro: Guanabara Koogan, 2007. p. 102-105.

DUARTE, T. P.; ANDRADE, N. A. Enfrentando a mastectomia: análise dos relatos de mulheres mastectomizadas sobre questôes ligadas a sexualidade. Estudos de Psicologia, Natal, v. 34, n. 1, p. 155-163, 2003. http://dx.doi.org/10.1590/S1413-294X2003000100017

EYSSENET, I. et al. The reproducibility of the canadian occupational performance measure. Clinical Rehabilitation, Oxford, v. 19, n. 8, p. 888-894, 2005. http://dx.doi. org/10.1191/0269215505cr883oa

INSTITUTO NACIONAL DE CÂNCER - INCA. Cancer de mama. Rio de Janeiro: INCA, 2008. Disponível em: <http://wwwl.inca.gov.br/inca/Arquivos/ relatorio_2008_2009.pdf>. Acesso em: 23 set. 2011.

LEITE, F. M. C. et al. Mulheres com diagnóstico de câncer de mama em tratamento com tamoxifeno: perfil sociodemográfico e clínico. Revista Brasileira de Cancerologia, Rio de Janeiro, v. 57, n. 1, p. 15-21, 2012.

KEBBE, L. M. Desempenho de atividades e imagem corporal: representaçóes sociais de um grupo de mulheres com câncer de mama. 2006. 158 f. Tese (Doutorado em Enfermagem em Saúde Pública)-Escola de Enfermagem de Ribeirão Preto, Universidade de São Paulo, Ribeirão Preto, 2006.
LAW, M. et al. (Org.). Manual de medida canadense de desempenho ocupacional (COPM). Belo Horizonte: UFMG, 2009.

MANUEL, J. et al. Perfil de pacientes submetidas à reconstrução mamária tardia atendidas em hospital universitário do município de São Paulo. Saúde Coletiva, São Paulo, v. 7, n. 39, p. 82-86, 2010.

MCCOLL, M. et al. Targeted applications of the canadian occupational performance measure. Canadian Journal of Occupational Therapy, Ottawa, v. 72, n. 5, p. 298-300, 2005. http://dx.doi.org/10.1177/000841740507200506

MELLO, M. A. F.; MANCINI, M. C. Avaliação das atividades de vida diária e controle domiciliar. In: CAVALCANTI, A.; GALVÂO, C. Terapia Ocupacional: fundamentos e prática. Rio de Janeiro: Guanabara Koogan, 2007. p. 49-54.

MISTURA, C.; CARVALHO, M. F. A. A.; SANTOS, V. E. P. S. Mulheres mastectomizadas: vivências frente ao câncer de mama. Revista de Enfermagem da UFSM, Santa Maria, v. 1, n. 3, p. 351-359, 2011.

OLIVEIRA, M. M. F. et al. Exercícios para membros superiores durante radioterapia para câncer de mama e qualidade de vida. Revista Brasileira de Ginecologia e Obstetrícia, Rio de Janeiro, v. 32, n. 3, p. 133-138, 2010.

OTHERO, M. B. Terapia ocupacional: práticas em oncologia. Sáo Paulo: Roca, 2010.

PARKER, D.; SYKES, C. A Systematic review of the canadian occupational performance measure: a clinical practice perspective. The British Journal of Occupational Therapy, London, v. 69, n. 4, p. 150-160, 2006.

PANOBIANCO, M. S.; MAMEDE, M. V. Complicaçôes e intercorrências associadas ao edema de braço nos três primeiros meses pós-mastectomia. Revista LatinoAmericana de Enfermagem, Ribeirão Preto, v. 10, n. 4, p. 544-551, 2002. http://dx.doi.org/10.1590/ S0104-11692002000400012

SASAKI, T.; LAMARI, N. M. Reabilitação funcional precoce pós-mastectomia. HB Cientifica, São José do Rio Preto, v. 4, n. 2, p. 121-127, 1997.

SPALLA, R. P.; SANTO, F. H. E. De frente para o desconhecido: histórias vividas com câncer. Revista Enfermagem Atual, Rio de Janeiro, v. 53, n. 9, p. 29-32, 2009.

SILVA, C. B.; ALBUQUERQUE, V.; LEITE, J. Qualidade de vida em pacientes portadoras de neoplasia mamária submetidas a tratamentos quimioterápicos. Revista Brasileira de Cancerologia, Rio de Janeiro, v. 56, n. 2, p. 227-236, 2010.

SILVA, P. A.; RIUL, S. S. Câncer de mama: fatores de risco e detecção precoce. Revista Brasileira de Enfermagem, Brasília, v. 64, n. 6, p. 1016-1021, 2011. http://dx.doi. org/10.1590/S0034-71672011000600005

TOLDRÁ, R.C. et al. Facilitadores e barreiras para o retorno ao trabalho. Revista Brasileira de Saúde Ocupacional, São Paulo, v. 35, n. 121, p. 10-22, 2010. http://dx.doi. org/10.1590/S0303-76572010000100003 


\section{Contribuição dos Autores}

Jamylle Brito foi responsável pela pesquisa e redação do artigo. Juliana Marcelino foi orientadora do trabalho. Ambas as autoras aprovaram a versão final do texto.

\section{Notas}

${ }^{1}$ Artigo científico apresentado ao Programa de Residência Multiprofissional Integrada em Saúde (PRMIS) do Hospital das Clínicas da Universidade Federal de Pernambuco (UFPE), como critério para obtenção do Título de Especialista em Terapia Ocupacional em Saúde da Mulher. Este estudo cumpriu os princípios éticos da Resolução do Conselho Nacional de Saúde, aprovado no Comitê de Ética e Pesquisa em Seres Humanos do Centro de Ciências da Saúde da UFPE sob o registro CAAE: 0107.0.172.000-11. 